\title{
1. Supplementary Data and Results
}

Table S1. Results from hierarchical piecewise structural equation modeling fitted using maximum likelihood. $B$, coefficient; $\mathrm{DF}$, degrees of freedom; $P, P$-value; $\mathrm{SE}$, standard error.

\begin{tabular}{|c|c|c|c|c|c|c|c|}
\hline Response & $\begin{array}{l}\text { Predictor } \\
\text { variables }\end{array}$ & $B$ & B SE & DF & $\begin{array}{r}\text { Critical } \\
\text { value }\end{array}$ & $P$ & Standardized $B$ \\
\hline \multirow{8}{*}{$\stackrel{\bar{s}}{ \pm 0}$} & Forest cover & 0.0218 & 0.00 & 12806 & 10.05 & 0.00 & 0.124 \\
\hline & Grassland cover & 0.0139 & 0.00 & 12806 & 6.47 & 0.00 & 0.067 \\
\hline & Cropland cover & -0.0068 & 0.00 & 12806 & -2.73 & 0.01 & -0.024 \\
\hline & $r_{\text {pasture }}$ & -0.0368 & 0.01 & 12806 & -5.89 & 0.00 & -0.051 \\
\hline & Conflict & -0.0016 & 0.00 & 12806 & -0.47 & 0.64 & -0.004 \\
\hline & Infrastructure & -0.0075 & 0.00 & 12806 & -1.83 & 0.07 & -0.019 \\
\hline & Municipality area & -0.0421 & 0.01 & 914 & -7.72 & 0.00 & -0.110 \\
\hline & Year & 0.0022 & 0.00 & 12806 & 4.96 & 0.00 & 0.050 \\
\hline \multirow{12}{*}{ 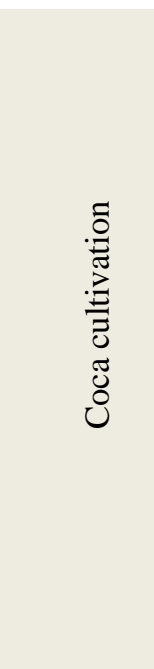 } & Forest cover & 0.0606 & 0.01 & 12802 & 4.59 & 0.00 & 0.078 \\
\hline & Grassland cover & -0.0666 & 0.01 & 12802 & -5.75 & 0.00 & -0.073 \\
\hline & Cropland cover & -0.1322 & 0.01 & 12802 & -9.95 & 0.00 & -0.105 \\
\hline & $r_{\text {pasture }}$ & 0.0233 & 0.01 & 12802 & 2.77 & 0.01 & 0.007 \\
\hline & $r_{\text {cropland }}$ & 0.0493 & 0.01 & 12802 & 5.01 & 0.00 & 0.013 \\
\hline & Infrastructure & -0.0136 & 0.01 & 12802 & -2.45 & 0.01 & -0.008 \\
\hline & Rural proportion & 0.3572 & 0.07 & 12802 & 5.16 & 0.00 & 0.097 \\
\hline & $r_{\text {urban population }}$ & 1.8609 & 0.32 & 12802 & 5.83 & 0.00 & 0.034 \\
\hline & $r_{\text {coca }}$ & 0.2231 & 0.00 & 12802 & 61.13 & 0.00 & 0.151 \\
\hline & Aerial fumigation & 0.1354 & 0.01 & 12802 & 27.10 & 0.00 & 0.124 \\
\hline & Manual eradication & 0.0535 & 0.00 & 12802 & 11.10 & 0.00 & 0.038 \\
\hline & Municipality area & 0.7505 & 0.04 & 914 & 17.13 & 0.00 & 0.444 \\
\hline \multirow{11}{*}{ 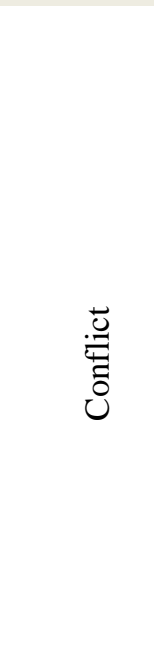 } & Forest cover & 0.073 & 0.01 & 12802 & 8.94 & 0.00 & 0.158 \\
\hline & Cropland cover & 0.0535 & 0.01 & 12802 & 4.72 & 0.00 & 0.071 \\
\hline & Infrastructure & 0.0167 & 0.01 & 12802 & 1.67 & 0.10 & 0.016 \\
\hline & $\begin{array}{l}\text { Total rural } \\
\text { population }\end{array}$ & 0.3832 & 0.02 & 12802 & 15.69 & 0.00 & 0.274 \\
\hline & Rural proportion & -0.5309 & 0.04 & 12802 & -14.40 & 0.00 & -0.241 \\
\hline & $r_{\text {urban population }}$ & -1.2935 & 0.39 & 12802 & -3.28 & 0.00 & -0.040 \\
\hline & $r_{\text {coca }}$ & -0.0245 & 0.01 & 12802 & -4.02 & 0.00 & -0.028 \\
\hline & Coca cultivation & 0.0551 & 0.01 & 12802 & 5.37 & 0.00 & 0.092 \\
\hline & Aerial fumigation & 0.0385 & 0.01 & 12802 & 5.08 & 0.00 & 0.059 \\
\hline & $\begin{array}{l}r_{\text {rural proportion }} \\
\text { Manual eradication }\end{array}$ & -0.0246 & 0.01 & 12802 & -3.36 & 0.00 & -0.029 \\
\hline & Year & -0.0213 & 0.001 & 12802 & -21.66 & 0.00 & -0.179 \\
\hline
\end{tabular}


Table S2. Results from hierarchical Bayesian structural equation modeling fitted using brms. B, coefficient mean; high probability density intervals (percent); SD, standard deviation; potential scale reduction factor (PSRF), and effective sample size (ESS).

\begin{tabular}{|c|c|c|c|c|c|c|c|}
\hline Response & Parameter & $B$ & $B$ SD & $2.50 \%$ & $97.50 \%$ & PSRF & ESS \\
\hline$r_{\text {forest }}$ & Intercept & -0.007 & 0.006 & -0.017 & 0.004 & 1.00 & 854 \\
\hline Coca cultivation & Intercept & -32.946 & 4.864 & -45.014 & -27.343 & 1.01 & 531 \\
\hline Conflict & Intercept & -15.147 & 4.777 & -27.019 & -9.784 & 1.02 & 550 \\
\hline$r_{\text {forest }}$ & Forest cover & -0.002 & 0.000 & -0.002 & -0.001 & 1.01 & 889 \\
\hline$r_{\text {forest }}$ & Grassland cover & 0.001 & 0.000 & 0.000 & 0.001 & 1.00 & 818 \\
\hline$r_{\text {forest }}$ & Cropland cover & 0.000 & 0.000 & -0.001 & 0.001 & 1.00 & 1050 \\
\hline$r_{\text {forest }}$ & $r_{\text {pasture }}$ & -0.008 & 0.001 & -0.010 & -0.006 & 1.00 & 1080 \\
\hline$r_{\text {forest }}$ & Conflict & -0.001 & 0.000 & -0.002 & 0.000 & 1.00 & 820 \\
\hline$r_{\text {forest }}$ & Infrastructure & 0.000 & 0.001 & -0.001 & 0.001 & 1.00 & 940 \\
\hline$r_{\text {forest }}$ & Municipality area & 0.001 & 0.001 & -0.001 & 0.002 & 1.01 & 867 \\
\hline Coca cultivation & Forest cover & 1.239 & 0.049 & 1.146 & 1.338 & 1.00 & 1026 \\
\hline Coca cultivation & Grassland cover & -0.532 & 0.058 & -0.651 & -0.416 & 1.00 & 953 \\
\hline Coca cultivation & Cropland cover & -0.142 & 0.051 & -0.238 & -0.037 & 1.00 & 988 \\
\hline Coca cultivation & $r_{\text {pasture }}$ & 0.894 & 0.137 & 0.620 & 1.173 & 1.00 & 864 \\
\hline Coca cultivation & $r_{\text {croplands }}$ & 0.206 & 0.170 & -0.131 & 0.532 & 1.00 & 969 \\
\hline Coca cultivation & Infrastructure & -0.528 & 0.093 & -0.721 & -0.356 & 1.00 & 862 \\
\hline Coca cultivation & Rural proportion & 2.806 & 0.169 & 2.474 & 3.137 & 1.00 & 1024 \\
\hline Coca cultivation & $r_{\text {urban population }}$ & 21.148 & 2.334 & 16.615 & 25.544 & 1.00 & 897 \\
\hline Coca cultivation & $r_{\text {соса }}$ & 0.819 & 0.036 & 0.747 & 0.891 & 1.00 & 996 \\
\hline Coca cultivation & Aerial fumigation & 0.974 & 0.042 & 0.895 & 1.053 & 1.00 & 893 \\
\hline Coca cultivation & Manual eradication & 1.245 & 0.063 & 1.125 & 1.370 & 1.00 & 995 \\
\hline Coca cultivation & Municipality area & 2.743 & 0.132 & 2.487 & 3.000 & 1.00 & 962 \\
\hline Conflict & Forest cover & 0.638 & 0.034 & 0.567 & 0.701 & 1.00 & 770 \\
\hline Conflict & Cropland cover & 0.752 & 0.050 & 0.656 & 0.851 & 1.00 & 979 \\
\hline Conflict & Infrastructure & 0.091 & 0.073 & -0.050 & 0.229 & 1.00 & 953 \\
\hline Conflict & Total rural population & 2.026 & 0.101 & 1.827 & 2.220 & 1.00 & 927 \\
\hline Conflict & Rural proportion & -3.133 & 0.130 & -3.398 & -2.888 & 1.00 & 1069 \\
\hline Conflict & $r_{\text {urban }}$ population & -16.766 & 2.047 & -20.944 & -12.695 & 1.00 & 956 \\
\hline Conflict & $r_{\text {coca }}$ & -0.069 & 0.047 & -0.156 & 0.028 & 1.00 & 666 \\
\hline Conflict & Coca cultivation & 0.736 & 0.060 & 0.618 & 0.854 & 1.00 & 928 \\
\hline Conflict & Aerial fumigation & 0.169 & 0.063 & 0.045 & 0.293 & 1.00 & 894 \\
\hline Conflict & Manual eradication & -0.091 & 0.061 & -0.207 & 0.028 & 1.00 & 883 \\
\hline$r_{\text {forest }}$ & sample-wide smoothing 1 & 0.000 & 0.001 & -0.001 & 0.001 & 1.00 & 919 \\
\hline$r_{\text {forest }}$ & sample-wide smoothing 2 & -0.003 & 0.001 & -0.004 & -0.002 & 1.00 & 971 \\
\hline
\end{tabular}




\begin{tabular}{|c|c|c|c|c|c|c|c|}
\hline$r_{\text {forest }}$ & sample-wide smoothing 3 & 0.000 & 0.000 & -0.001 & 0.001 & 1.00 & 815 \\
\hline Coca cultivation & sample-wide smoothing 1 & 137.099 & 102.923 & 28.397 & 406.449 & 1.00 & 519 \\
\hline Coca cultivation & sample-wide smoothing 2 & -0.148 & 2.716 & -5.667 & 4.662 & 1.00 & 760 \\
\hline Coca cultivation & sample-wide smoothing 3 & -1.292 & 58.900 & -124.651 & 104.881 & 1.00 & 742 \\
\hline Conflict & sample-wide smoothing 1 & 154.245 & 102.712 & 41.682 & 410.536 & 1.01 & 578 \\
\hline Conflict & sample-wide smoothing 2 & 1.474 & 2.915 & -4.422 & 7.374 & 1.00 & 810 \\
\hline Conflict & sample-wide smoothing 3 & 3.535 & 63.331 & -126.187 & 128.459 & 1.00 & 811 \\
\hline$r_{\text {forest }}$ & $\begin{array}{l}\text { group-specific smooth } \\
\text { sigma } 1\end{array}$ & 0.539 & 0.196 & 0.264 & 1.068 & 1.00 & 784 \\
\hline$r_{\text {forest }}$ & $\begin{array}{l}\text { group-specific smooth } \\
\text { sigma } 2\end{array}$ & 0.804 & 0.331 & 0.362 & 1.663 & 1.00 & 899 \\
\hline$r_{\text {forest }}$ & $\begin{array}{l}\text { group-specific smooth } \\
\text { sigma } 3\end{array}$ & 0.100 & 0.097 & 0.004 & 0.314 & 1.00 & 862 \\
\hline Coca cultivation & $\begin{array}{l}\text { group-specific smooth } \\
\text { sigma } 1\end{array}$ & 101.354 & 25.854 & 63.085 & 164.439 & 1.00 & 806 \\
\hline Coca cultivation & $\begin{array}{l}\text { group-specific smooth } \\
\text { sigma } 2\end{array}$ & 2.897 & 3.627 & 0.114 & 11.019 & 1.00 & 809 \\
\hline Coca cultivation & $\begin{array}{l}\text { group-specific smooth } \\
\text { sigma } 3\end{array}$ & 138.391 & 57.899 & 64.036 & 276.297 & 1.00 & 1026 \\
\hline Conflict & $\begin{array}{l}\text { group-specific smooth } \\
\text { sigma } 1\end{array}$ & 71.203 & 21.560 & 38.685 & 119.239 & 1.00 & 813 \\
\hline Conflict & $\begin{array}{l}\text { group-specific smooth } \\
\text { sigma } 2\end{array}$ & 95.200 & 31.610 & 51.917 & 168.802 & 1.00 & 851 \\
\hline Conflict & $\begin{array}{l}\text { group-specific smooth } \\
\text { sigma } 3\end{array}$ & 138.977 & 56.299 & 64.814 & 272.810 & 1.00 & 879 \\
\hline$r_{\text {forest }}$ & $\begin{array}{l}\text { sample-wide standard } \\
\text { deviation }\end{array}$ & 0.021 & 0.000 & 0.020 & 0.021 & 1.00 & 1106 \\
\hline$r_{\text {forest }}$ & $\mathrm{Nu}$ & 1.001 & 0.001 & 1.000 & 1.002 & 1.00 & 1032 \\
\hline Coca cultivation & gamma-Poisson shape & 0.089 & 0.002 & 0.085 & 0.093 & 1.00 & 959 \\
\hline Conflict & gamma-Poisson shape & 0.094 & 0.002 & 0.090 & 0.097 & 1.00 & 855 \\
\hline Whole model & log posterior density & -27581 & 10 & -27601 & -27563 & 1.00 & 675 \\
\hline
\end{tabular}



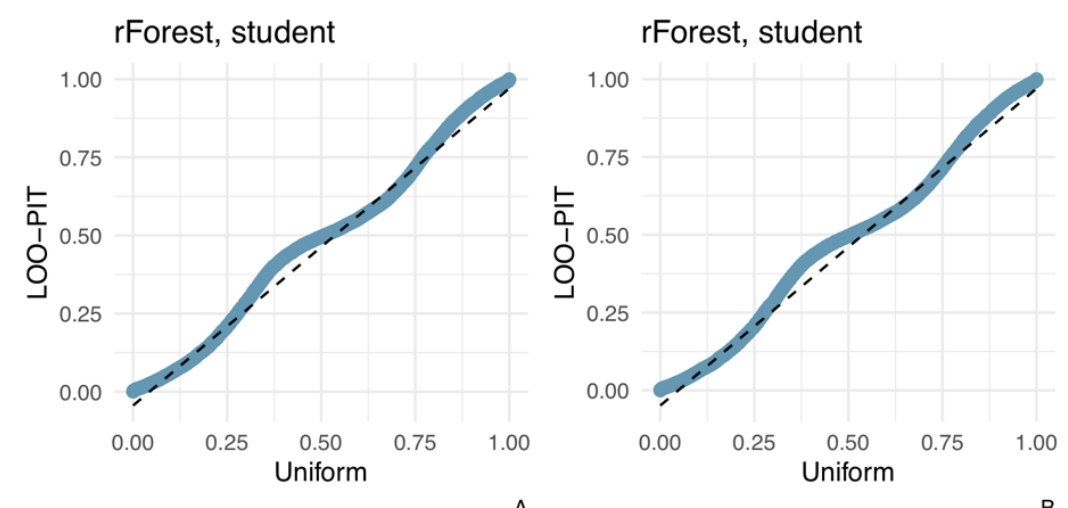

A

B

Coca cultivation, negative binomial Coca cultivation, zero-inflated
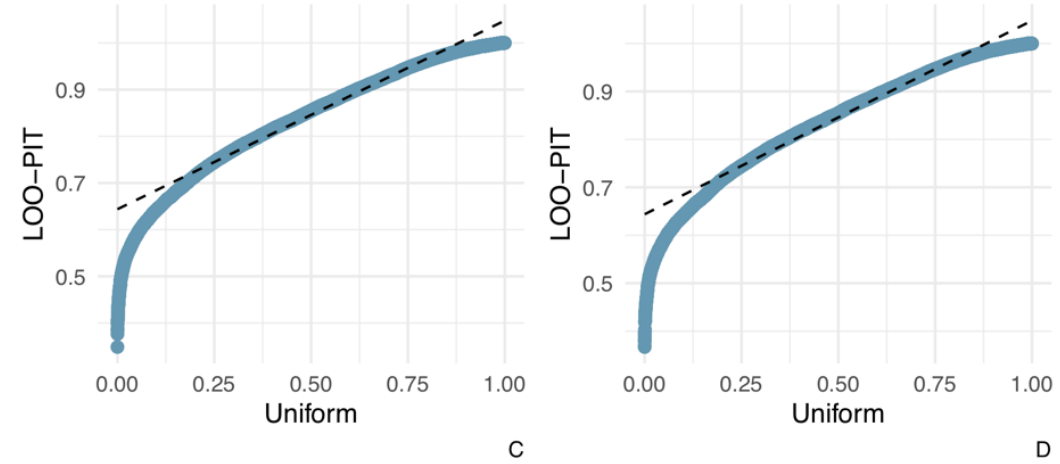

Conflict, negative binomial

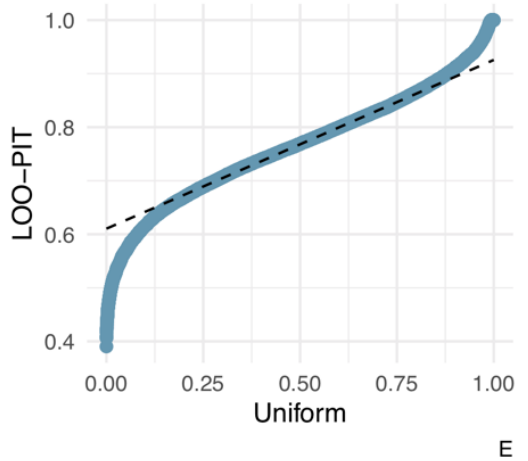

Conflict, zero-inflated

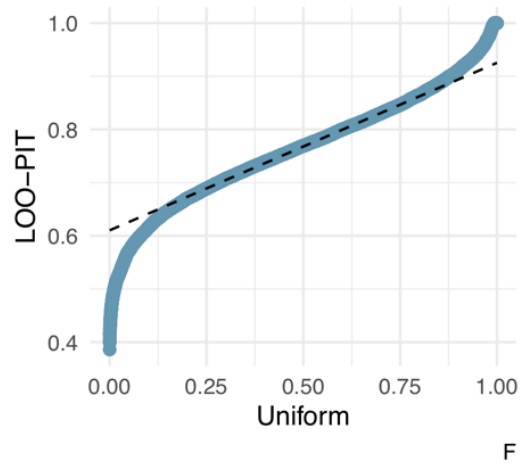

Figure S1. Posterior predictive checks using leave-one-out probability integral transformations (loo-pit) on multivariate models of $r_{\text {forest }}$ as a Student's $t$-distributed variable (i.e., with 'fat' tails and leptokurtotic center; A and B), and of coca cultivation (C and D) and conflict (E and F) victim counts as negative binomial (gamma-Poisson; C and E), or zero-inflated negative binomial (D and f) distributions. Note: Models applied Markov random fields smoothing (MRF) 
to account for spatiotemporal trends and modeled. In these quantile-quantile plots, wellcalibrated model values line up along the dashed line.
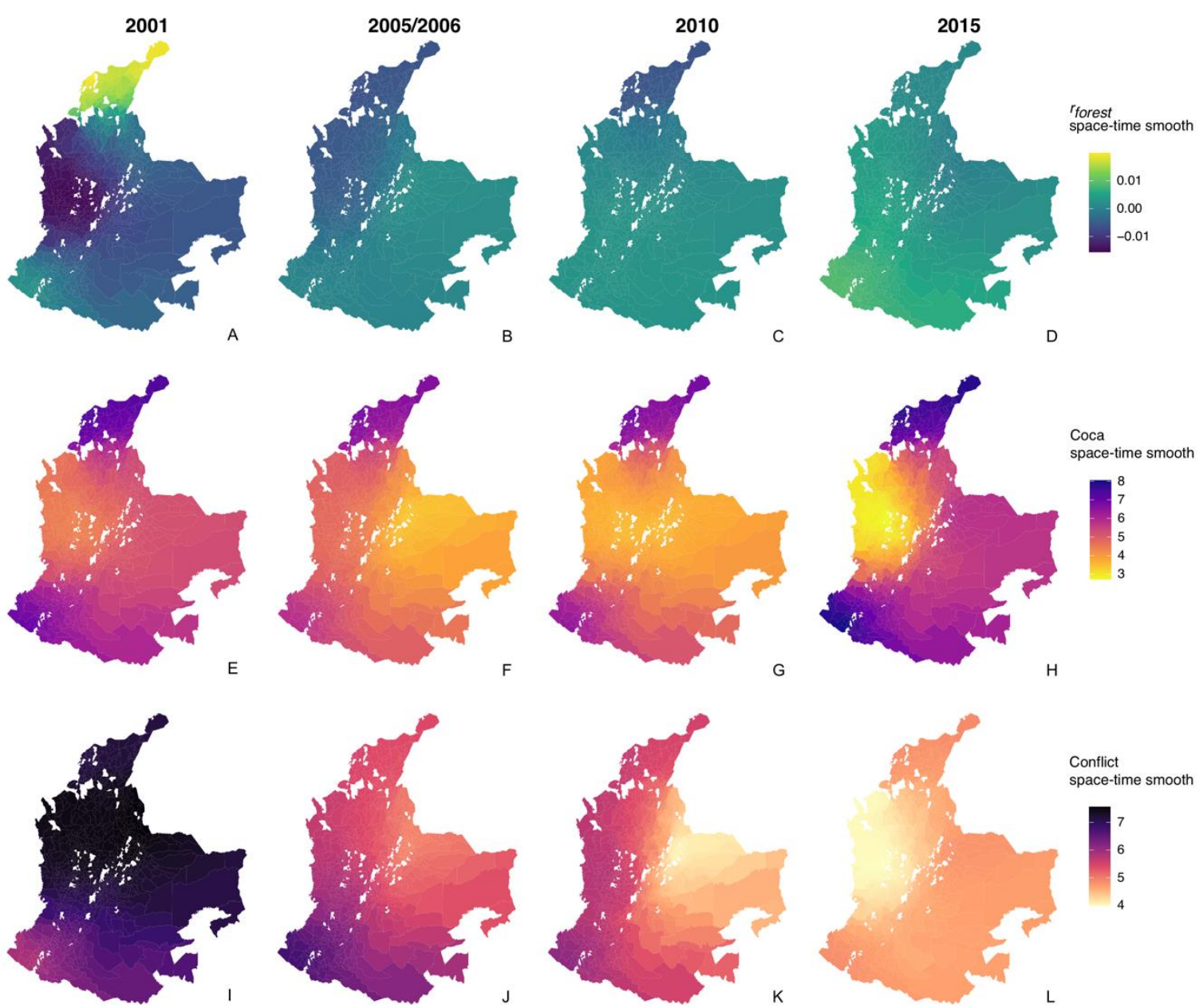

Figure S2. Spatiotemporal smooths (i.e., spatiotemporal trends but not the data itself) from fitting Gaussian Markov random fields (GMRF) to the $r_{\text {forest }}(\mathrm{A}-\mathrm{D})$, coca cultivation (E-H), and conflict (I-L) data, for Colombia in 2001 (A, E, and I) 2005/2006 (B, F, and J), 2010 (C, G, and K), and 2015 (D, H, and L)). Note: Two municipalities, Puerto Nariño and Leticia in the department of Amazonas, were omitted from these visualizations as their low coca and conflict counts made color changes less visible. For consistency, these were also omitted from the $r_{\text {forest }}$ plots. 